\title{
Relação dose-resposta entre nível de atividade física e desfechos em saúde
}

\author{
Dose-response relationship between physical activity and health outcomes
}

\author{
Alice Ricciardi Sampaio \\ Jonathan Myers \\ Ricardo B. Oliveira ${ }^{\star}$
}

\section{Resumo}

A compreensão sobre a relação de doseresposta entre atividade física e saúde se faz necessária, em uma perspectiva de saúde pública. O estudo das relações de dose-resposta entre atividade física e saúde deve considerar a interação entre os diversos componentes da atividade física, como o volume (frequência e duração) e a intensidade. Apesar de resultados conflitantes, evidências sugerem uma relação inversa de dose-resposta entre o volume total de atividade física e risco de mortalidade por todas as causas para ambos os sexos, entre jovens adultos e idosos que despendem ao menos 500 $\mathrm{kcal} / \mathrm{semana}$ (idealmente ao menos $1.000 \mathrm{kcal} /$ semana), que caminham ao menos de 1 a $3 \mathrm{~km}$ por dia de modo contínuo ou acumulado em sessões de pelo menos 10 a 15 minutos, com reduções de risco na ordem de $20 \%$ a $30 \%$. Até a presente data, são escassos os dados da literatura capazes de estabelecer com clareza a forma da curva de dose-resposta entre a intensidade da atividade física e risco de mortalidade. Em parte, devido a algumas limitações inerentes aos estudos observacionais, como a dificuldade em se quantificar de modo preciso, os níveis de atividade física nos indivíduos em situação de vida real. Futuros estudos deverão incorporar o uso de novas tecnologias para a avaliação dos hábitos de atividade física, bem como serão capazes de gerenciar e analisar a grande quantidade de dados que essas novas tecnologias permitirão coletar, de modo a melhor esclarecer as inter-relações entre os diversos componentes da atividade física e diversas doenças.

Descritores: Epidemiologia; Saúde; Aptidão física; Fatores de risco; Exercício físico.

\section{Abstract}

In a public health perspective, it is important to understand the relationship between the dose-response curve of physical activity and health outcomes. Studies aiming to investigate such relationships must consider the inter-relationship among the components of 
physical activity as the volume (frequency and duration) and intensity. Evidence suggests an inverse dose-response between the total volume of physical activity and all-cause mortality risk in both sex, among young adulthood and older subjects who spend at least $500 \mathrm{kcal} /$ week (ideally at least $1000 \mathrm{kcal} /$ week), walk 1 to 2 miles/day continuously or in short bouts of 10 to 15 minutes along the day, showing a risk reduction of about $20 \%$ to $30 \%$. To date, no clear data exist regarding the dose-response shape of the curve between the intensity of physical activity and the risk of clinical events, including all-cause mortality. This may be explained by the difficulties faced by researchers in precisely quantifying physical activity behavior in real life conditions. Future studies should be able to incorporate new technologies that will challenge investigators, considering the enormous set of data that will be probably gathered almost in real-time and therefore needed to be analyzed properly.

Keywords: Epidemiology; Health; Physical fitness; Risk factors; Physical exercise.

\section{Introdução}

Desde 2500 a.C., na China antiga, existem relatos sobre o uso planejado da atividade física como forma de promoção da saúde. ${ }^{1}$ Hoje, há dados convincentes de que uma vida ativa pode trazer benefícios à saúde e que hábitos sedentários estariam associados a aumento nos riscos de doenças crônico-degenerativas., ${ }^{2,3}$ Embora a relação entre a prática de atividade física e saúde esteja clara, consensos sobre a dose específica necessária para atingir tais benefícios, ainda permanecem vagos. ${ }^{4}$

A compreensão sobre a relação de dose-resposta entre atividade física e saúde se faz necessária, na medida em que recomendações de quantidades mínimas, adequadas às demandas da população, possam ser atendidas. A falta de um consenso sobre a ótima relação de dose-resposta evidencia-se pelas divergentes recomendações preconizadas por algumas das principais diretrizes de agências institucionais normativas. ${ }^{2,5}$ Recomendações preconizadas entre os anos de 1970 e 1980 destacavam a importância da realização de exercícios vigorosos por, no mínimo, 20 minutos contínuos, com frequência semanal de três dias. ${ }^{6}$ No entanto, a partir da década de 1990, o peso dado para atividades intensas foi retirado, mesmo reconhecendo-se a escassez de estudos avaliando os benefícios de exercícios de intensidade moderada. Assim, as recomendações passaram a preconizar atividades de intensidade moderada por 30 minutos, que poderiam ser acumulados ao longo do dia, realizados na maioria dos dias da semana. ${ }^{7}$ Atualmente, tais recomendações apontam para a realização de 150 minutos/semana de atividade com intensidade moderada ou 75 minutos/semana de atividade com intensidade vigorosa ou uma combinação equivalente (7,5 MET-hora/ semana) para benefícios à saúde.

Pode-se observar, portanto, uma falta de consenso histórico sobre a dose mínima adequada de atividade física. Em parte, a carência de consensos sobre a ótima relação de dose-resposta entre atividade física e saúde deriva das diversas limitações e divergências metodológicas enfrentadas por pesquisadores no campo da epidemiologia da atividade física.

Sendo assim, o objetivo desta revisão é discutir as relações de dose-resposta entre atividade física e saúde, com ênfase nas relações entre volume (duração e frequência) e intensidade (absoluta e relativa). Em adendo, a presente revisão discute algumas limitações metodológicas dos estudos nesse campo e aponta futuras perspectivas e abordagens metodológicas.

\section{Razões pelo interesse da dose- resposta}

Existem algumas razões pelas quais a relação de dose-resposta entre atividade física e saúde interessa tanto aos pesquisadores quanto a população. Primeiramente, a importância em sabermos sobre a relação de dose-resposta é 
fornecer o conhecimento da mínima quantidade de atividade física necessária para atingir benefícios à saúde, sendo esta informação traduzida como recomendação ao público. ${ }^{8}$ Considerando que a proporção de indivíduos que realizam atividades físicas no tempo de lazer, adequadas às atuais recomendações, giram em torno de $28 \%$ e $36 \%$ entre mulheres e homens, respectivamente, parece irreal criar expectativas de que a grande maioria dos indivíduos irá seguir recomendações em realizar atividades físicas vigorosas e de longa duração. ${ }^{3}$ A recomendação direcionada para quantidades modestas de atividade física, porém suficientes em fornecer benefícios à saúde, parece ser mais palatável para a grande maioria da população e precisam, portanto, ser mais bem-investigadas. ${ }^{9}$

Outra razão para o interesse em se investigar a relação de dose-resposta é saber se de fato existe uma relação real de causa e efeito entre a prática de atividade física e determinado desfecho clínico. Apesar de estudos observacionais não serem considerados metodologicamente robustos na determinação de relações de causa e efeito, diversos autores têm proposto algumas abordagens capazes de estabelecer tais relações. ${ }^{10}$ Apesar de estudos controlados e randomizados serem considerados ideais para o estabelecimento de relações de causa e efeito, os resultados de tais estudos por vezes são relativamente limitados quando aplicados em condições reais da população. Participantes que se voluntariam a participar de estudos controlados costumam ser mais saudáveis; ${ }^{11}$ além disso, o número limitado de grupos comparados, muitas vezes não dá conta de explorar as diferentes características da atividade física, como o tipo de atividade, as diferentes intensidades, frequências e durações. Estudos observacionais, ao explorar as relações de causa e efeito, podem adicionar, portanto, informação relevante que muitas vezes não podem ser respondidas por ensaios clínicos controlados e randomizados.

Uma terceira razão pelo interesse da relação de dose-resposta é o levantamento de hipóteses, que, através das associações estabelecidas em modelos multivariados, são capazes de indicar potenciais mecanismos fisiológicos a serem posteriormente investigados em estudos controlados e randomizados. ${ }^{12}$

\section{Inter-relações entre os componentes da atividade física}

Em 2000, o Instituto de Saúde Canadense e o Centro de Controle e Prevenção de Doenças dos Estados Unidos (CDC) organizaram um simpósio científico, estabelecendo que o estudo das relações entre atividade física e saúde deveria considerar os diversos componentes da atividade física, como a frequência, a duração e a intensidade da atividade física. ${ }^{4}$ No entanto, as inter-relações entre tais componentes da atividade física contribuem para a complexidade das análises de dose-resposta. Para muitos autores, a relação de dose-resposta é investigada ao comparar grupos estratificados em função do volume total de atividade física, comumente expresso em gasto calórico total (kcal/semana) ou múltiplos de equivalente metabólico por tempo (MET/hora). ${ }^{8}$

$\mathrm{Na}$ medida em que atividades físicas de maior intensidade, duração e frequência, levam a um maior dispêndio energético ou gasto calórico total, torna-se difícil determinar até que ponto os benefícios das atividades são derivados pelo simples aumento do dispêndio energético ou exclusivamente pelo aumento de cada um desses componentes de modo independente. Tal fato torna-se ainda mais complexo, no momento em que muitos autores quantificam as atividades físicas pelos valores de METs associados a cada atividade física realizada. Ao assumir que 1 MET equivale a 3,5 $\mathrm{ml} \cdot \mathrm{kg}^{-1} \cdot \mathrm{min}^{-1}$ ou $5 \mathrm{kcal}$, esta classificação dificulta ainda mais a separação da intensidade e do volume total realizado, uma vez que o valor do METs normalmente é utilizado para quantificar ambos os componentes.

Para uma melhor compreensão da dose mínima de atividade física e sua relação com 
saúde, abordagens metodológicas precisam ser capazes de isolar de modo independente a contribuição de cada um dos componentes. Assim, algumas questões precisam ser consideradas:

1) Qual a dose mínima de intensidade associada à redução de risco de doenças crônicas e mortalidade por todas as causas? Mantendo a duração e a frequência constantes, o aumento da intensidade leva a uma redução linear dos riscos ou existe um ponto no qual aumentos de intensidade não se associam a reduções adicionais de risco?

2) Qual a dose mínima de duração associada à redução de risco de doenças crônicas e mortalidade por todas as causas? Mantendo a intensidade e a frequência constantes, $o$ aumento da duração leva a uma redução linear dos riscos?

3) Qual a frequência mínima associada à redução de risco de doenças crônicas e mortalidade por todas as causas? Mantendo a intensidade e a duração constantes, $o$ aumento da frequência leva a uma redução linear dos riscos?

No presente estudo, para a melhor compreensão do tema, o volume da atividade física (duração e frequência) e a intensidade (relativa e absoluta) serão considerados de modo separado.

\section{Forma da curva de dose- resposta}

Apesar da informação consistente de que a atividade física fornece benefícios à saúde, ainda não é tão clara a forma da curva de dose-resposta entre atividade física e diferentes desfechos clínicos. ${ }^{4}$ No entanto, podemos possivelmente assumir que a quantidade mínima de atividade física necessária para se atingir benefícios à saúde difere em função do desfecho analisado.

Segundo as recomendações do CDC e do Colégio Americano de Medicina do Esporte (ACSM), 30 minutos diários de atividade moderada parecem ser capazes de reduzir o risco de desenvolvimento de doenças cardiovasculares principalmente entre mulheres, idosos e sedentários. ${ }^{3,13,14}$ Por outro lado, pesquisadores sugerem que maiores volumes de atividade física são necessários para mudanças benéficas em certas lipoproteínas do sangue, ${ }^{15}$ e também para a manutenção de um peso saudável de indivíduos previamente obesos. ${ }^{16}$ Devido à etiologia multifatorial de desfechos clínicos, como a doença arterial coronariana, é natural de se esperar que a relação de dose-resposta entre atividade física e um único fator de risco não seja exatamente a mesma do que entre a atividade física e determinado desfecho clínico.

Além da importância em se conhecer a quantidade mínima capaz de promover benefícios à saúde, também é fundamental conhecermos a forma da curva de doseresposta acima das quantidades mínimas de atividade física. Na figura 1 observam-se três possíveis comportamentos das relações de dose-resposta de atividade física de desfechos clínicos. Caso determinado desfecho clínico apresente o comportamento representado pela curva $\mathrm{A}$, poderíamos afirmar que cada incremento de atividade física estaria associado a um benefício adicional de modo linear. No entanto, caso a curva de dose-resposta apresente um comportamento ilustrado pela curva $\mathrm{B}$, consequentemente, grandes benefícios ocorrerão para pequenos aumentos nos hábitos de atividade física. Finalmente, caso a curva $\mathrm{C}$ seja a observada, benefícios adicionais seriam observados apenas com grandes aumentos nos hábitos de atividade física.

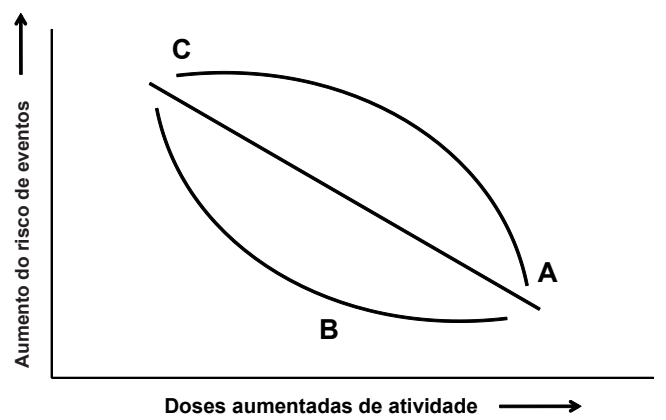

Figura 1. Curvas de dose-resposta de atividade física e desfechos em saúde 
Apesar dos avanços observados na epidemiologia da atividade física, desde o estudo pioneiro do Professor Jeremy Morris, no ano de $1953,{ }^{17}$ ainda são poucos os dados na literatura capazes de determinar a forma da curva de dose-resposta entre atividade física e saúde. Um dos maiores desafios diz respeito à comparação dos resultados dos diferentes estudos, na medida em que diferentes classificações e instrumentos de avaliação da atividade física tornam praticamente impossível a conversão dos dados em uma única escala de comparação.

Considerando algumas das questões mencionadas, na próxima sessão da presente revisão, serão discutidos estudos que receberam maior destaque na comunidade científica internacional e que tiveram como preocupação a inter-relação entre os componentes da atividade física, de modo que os mesmos sejam apresentados e analisados de forma independente.

\section{Dose-resposta para o volume de atividade física}

O volume total de atividade física é o componente mais simples e amplamente investigado, de modo que a maior parte do conhecimento ligado às relações de doseresposta é derivada deste componente. Isto se deve ao fato de que, independentemente do tipo, da duração, da frequência ou da intensidade da atividade física, de algum modo, todos estarão relacionados ao gasto energético despendido e consequentemente ao volume total de atividade realizado. Embora avaliações simples do volume de atividade física, como as realizadas no U.S. Longitudinal Study of Aging, ${ }^{18}$ em que grupos foram comparados através da percepção subjetiva de quão ativos eram em comparação aos seus pares de mesma idade, já sejam capazes de identificar uma relação inversa com a mortalidade (menos ativos apresentaram um risco $40 \%$ superior de mortalidade), tal abordagem não permite quantificar o papel independente de cada um dos componentes que integram o dispêndio energético total e, portanto, limita-se no que diz respeito à aplicabilidade para a saúde pública. De modo semelhante, dados do importante Harvard Alumni Healthy Study, ${ }^{19}$ apesar de quantificar o volume de atividade física em $\mathrm{kcal} / \mathrm{semana} \mathrm{e}$ revelar uma curva de dose-resposta em forma de "L", com as maiores reduções de risco sendo observadas entre os grupos dos três primeiros quintis, sem benefícios adicionais observados para volumes superiores a $1.999 \mathrm{kcal} /$ semana, também são limitados, na medida em que nesta análise específica, cada componente da atividade física não é tratado de modo independente, não respondendo, assim, a questões como: qual a duração mínima necessária para que efeitos benéficos à saúde sejam garantidos; sessões de curta duração (10 a 15 minutos) acumuladas ao longo do dia podem ser realizadas ou apenas sessões únicas de longa duração (30 a 60 minutos) são capazes de promover benefícios; ou em outras palavras, para dois indivíduos que despendem o mesmo gasto calórico, existe diferença entre atingir tais valores ao realizar uma única sessão de 30 minutos por dia durante cinco dias por semana ou realizar duas sessões diárias de 15 minutos?

Neste sentido, Lee e colaboradores, ${ }^{20} \mathrm{em}$ um dos poucos estudos que procurou responder a essas questões, os indivíduos relataram o tempo em que realizavam suas atividades esportivas e de lazer, sendo classificados em grupos em função da duração total gasta em suas atividades (sem atividade - grupo-referência -, 1-15; 16-30; 31-45; 46-60 e acima de 60 minutos). Quando ajustado pela idade e variáveis clínicas, o modelo multivariado revelou uma tendência a uma relação inversa de dose-resposta entre a duração total realizada e o risco de eventos coronarianos, em que apenas aqueles que realizavam atividades com duração superior a 60 minutos apresentaram redução de $22 \%$ de eventos, quando comparados ao gruporeferência $[0,78(0,62-0,98)]$. Tais resultados sugerem, portanto, que atividades de maiores durações seriam necessárias para que os benefícios fossem alcançados. No entanto, 
ao ajustar o modelo multivariado também pelo gasto calórico total realizado por cada indivíduo, a associação entre a duração da atividade física e o risco de eventos coronarianos não foi observada, sugerindo que conquanto o dispêndio energético seja suficientemente adequado (ver resultados anteriores), não importa a duração da atividade realizada. Estes resultados deram suporte às atuais recomendações do CDC/ACSM que sugerem que as atividades físicas possam ser acumuladas em sessões de curta duração (10 a 15 minutos) ao longo do dia. Até a presente data, apenas poucos estudos ${ }^{21-23}$ procuraram avaliar as associações entre a frequência das atividades físicas e taxas de mortalidade. No entanto, como em tais estudos os modelos multivariados não controlaram suas análises pelo gasto calórico despendido pelos indivíduos, é provável que tais resultados reflitam mais as associações já conhecidas entre o volume total realizado de atividades e os riscos de mortalidade.

Em síntese, apesar de ainda existirem resultados conflitantes na literatura, evidências sugerem uma relação inversa de dose-resposta

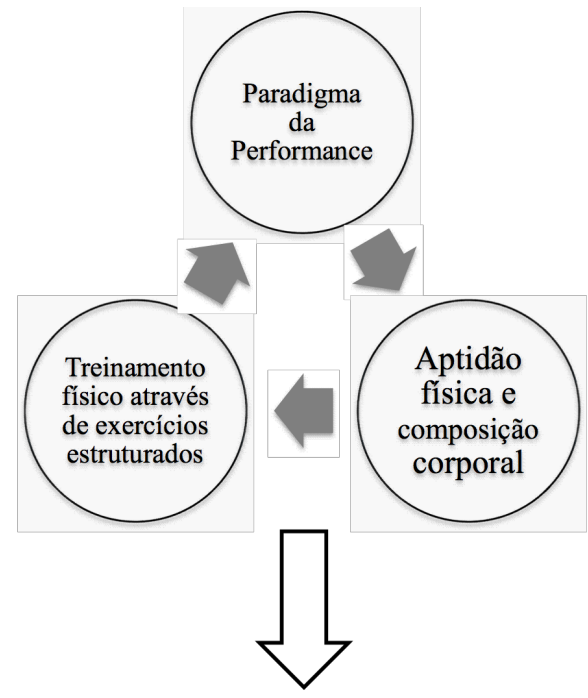

Atividades de endurance com duração de 20 a 60 minutos, 3 a 5 dias/semana, 60 a $90 \%$ da FCmáx ou 50 a $85 \%$ do $\mathrm{VO}_{2}$ máx ou da F. C. de reserva (> 6 METs). entre o volume total de atividade física e risco de mortalidade por todas as causas para ambos os sexos, entre jovens adultos e idosos que despendem ao menos $500 \mathrm{kcal} / \mathrm{semana}$ (idealmente ao menos $1.000 \mathrm{kcal} / \mathrm{semana}$ ), que caminham ao menos de 1 a $3 \mathrm{~km}$ por dia de modo contínuo ou acumulado em sessões de pelo menos 10 a 15 minutos. Reduções de risco na ordem de $20 \%$ a $30 \%$ são observadas.

\section{Dose-resposta para intensidade da atividade física}

As recomendações anteriores a 1995 do CDC/ACSM foram fundamentalmente baseadas em estudos no campo da fisiologia do exercício, principalmente, nas respostas agudas e adaptações crônicas ao exercício aeróbio para o aumento de desempenho - especialmente da aptidão cardiorrespiratória e da força muscular. O foco era voltado para exercícios de alta intensidade, como o trote e a corrida, uma vez que a alta intensidade associada a este tipo de atividade leva a adaptações cardiorrespiratórias maiores em curto prazo (Figura 2).

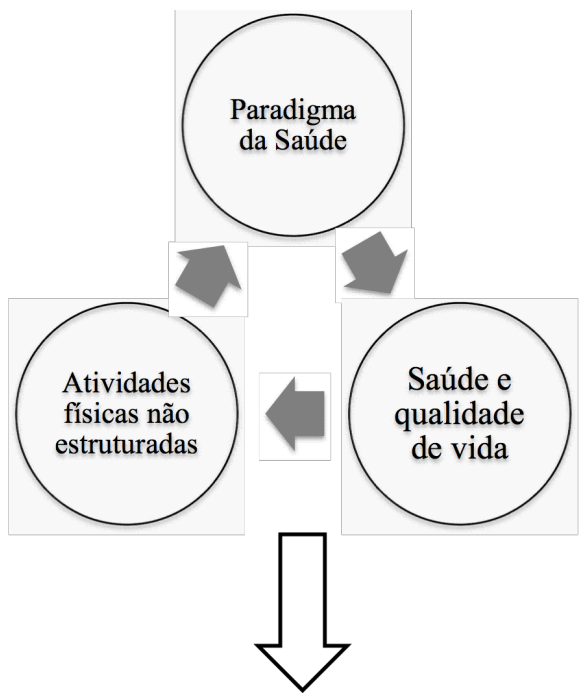

$\geq 30$ minutos de atividades contínuas ou intermitentes (sessões de 10 minutos ao longo do dia) com intensidade moderada (3-6 METs) na maioria dos dias da semana, preferencialmente todos os dias. 
A primeira recomendação de saúde pública entre atividade física e saúde foi de Pate e colaboradores, ${ }^{3}$ que incluía a realização de pelo menos 30 minutos de atividade física com intensidade moderada na maioria dos dias da semana. Dados, a partir de estudos experimentais e observacionais, demonstraram benefícios relacionados à saúde com atividades físicas moderadas acumuladas ao longo do dia. ${ }^{8}$ Esta recomendação partiu do consenso que o sedentarismo é um dos principais fatores de risco relacionado às diversas doenças crônicas ao longo da vida. ${ }^{8}$

A partir da recomendação de $1995,{ }^{3}$ houve grande consonância entre pesquisadores sobre a atividade física de intensidade moderada ser suficiente para se atingir benefícios à saúde. Já está claro na literatura que grandes volumes de gastos calóricos estão associados com menores incidências de doenças crônicas. ${ }^{8}$ Considerando a alta prevalência de sedentarismo e a baixa incidência de pessoas que engajam em atividades vigorosas, foi assumido que os gastos calóricos elevados oriundos desses estudos, seriam em sua maioria, de atividades com intensidade moderada. ${ }^{3}$

Estudos observacionais que têm por objetivo investigar as relações de dose-resposta entre a intensidade da atividade física e desfechos clínicos como mortalidade devem considerar comparações entre atividades de diferentes níveis de intensidade, considerando em seus modelos o volume total de gasto calórico nessas atividades. Um método de análise que pode avaliar a dose-resposta para a intensidade da atividade física, independentemente do volume energético total, é o uso de um modelo estatístico conhecido como modelo proporcional de risco ou modelo-padrão multivariado, comumente utilizado em estudos observacionais na área de nutrição. Neste modelo, os ajustes estimados excluem os efeitos das variáveis intervenientes e, com isso, podem ser feitos ajustes para o volume do gasto calórico total, e termos relacionados à intensidade da atividade física entram no modelo simultaneamente. ${ }^{24}$
Nas análises feitas por Tanasescu e colaboradores, ${ }^{25}$ além de ajustes por variáveis intervenientes relevantes, os pesquisadores ajustaram o modelo-padrão multivariado para o volume energético total, em MET-hora por semana. $\mathrm{O}$ estudo demonstrou uma relação inversa de dose-resposta entre a intensidade da atividade física e taxas de doença arterial coronariana, independentemente do fato de atividades vigorosas levarem a um maior gasto calórico. Atividades de intensidade moderada não apresentaram reduções importantes de risco quando comparadas às atividades de baixa intensidade.

Segundo Lee e colaboradores, ${ }^{26}$ ainda existem dados contraditórios na literatura no que diz respeito à intensidade moderada de atividade física e sua associação com a mortalidade. Em seu estudo, conduzido em ex-alunos (homens) de Harvard, grupos foram divididos tanto quanto ao gasto calórico total (< $150 \mathrm{kcal} /$ semana - grupo-referência -; 150-399 $\mathrm{kcal} / \mathrm{semana}$; 400-749 kcal/semana; 750-1.499 $\mathrm{kcal} /$ semana; $\geq 1.500$ ), como pela intensidade (vigorosa versus não vigorosa). Quando comparados ao grupo-referência, aqueles que realizaram atividades com maior gasto calórico, porém de intensidade moderada, não apresentaram reduções significativas de risco, diferentemente daqueles com maior volume, porém com intensidade vigorosa, sugerindo que atividades físicas de intensidades vigorosas seriam necessárias para a redução dos riscos de mortalidade. Uma explicação alternativa para os resultados, no entanto, pode residir no fato de que questionários, como no caso deste estudo, costumam não ser sensíveis a avaliar atividades de baixa e moderada intensidade, dificultando assim a correta classificação dos grupos. Outra possibilidade e eventual limitação do estudo é o fato de que no grupo de atividades não vigorosas foram incluídos todos os que realizavam atividades de leve a moderada intensidade. Desse modo, os possíveis efeitos das atividades de moderada intensidade podem ter sido diluídos pelos que realizavam atividades 
apenas de intensidade leve.

A metanálise de Löllgen ${ }^{27}$ revelou uma relação inversa entre a taxa de mortalidade por todas as causas e a intensidade com a qual as atividades físicas foram realizadas. Foram incluídos estudos em que pelo menos três categorias de intensidade fossem comparadas (nenhuma atividade - grupo-referência -, leve, moderada, vigorosa). As classificações das atividades físicas nos estudos analisados referem-se principalmente a kcal ou METs, sendo os sedentários aqueles com gasto calórico inferior a $200 \mathrm{kcal} / \mathrm{semana}(<1 \mathrm{MET})$, ligeiramente ativos com 200 a $599 \mathrm{kcal} / \mathrm{semana}$ (<3 METs), moderadamente ativos com 600 a $1.499 \mathrm{kcal} / \mathrm{semana}$ ( 3 a $6 \mathrm{METs}$ ) e vigorosamente ativos com mais de $1.500 \mathrm{kcal} / \mathrm{semana}$ (> 6 METs). Quando feitos os ajustes estimativos multivariados para atividades moderadas em homens (600 a $1.499 \mathrm{kcal} / \mathrm{semana}$ ), dos 11 estudos analisados, apenas cinco se mostraram estatisticamente significativos na redução de risco em comparação ao grupo sedentário. Enquanto que para as mulheres que realizaram atividades moderadas, dos sete estudos, quatro conferiram um resultado significativo. Nos ajustes estimativos multivariados para atividades vigorosas em homens (com mais de 1.500 $\mathrm{kcal} / \mathrm{semana}$ ), dos vinte estudos analisados, apenas cinco não revelaram um resultado estatisticamente significativo em comparação ao grupo sedentário. Sendo que para as mulheres, apenas dois estudos, dentro dos sete analisados, não demonstraram significância nos seus resultados. Quando comparados aos seus pares que realizavam atividades leves, homens e mulheres que realizavam atividades de intensidade alta apresentaram uma redução de risco de mortalidade por todas as causas de $22 \%$ e $31 \%$, respectivamente. Esses resultados foram semelhantes para aqueles que realizaram atividades de moderada intensidade, com reduções de 19\% e 24\% para homens e mulheres, respectivamente. Os autores concluem que intensidades mais elevadas são necessárias em uma perspectiva desportiva, mas não na prevenção primária.

Em estudo conduzido pelo nosso grupo, em colaboração com pesquisadores da Universidade de Stanford (dados ainda não publicados), as interações entre a aptidão cardiorrespiratória, o volume de atividade física e intensidade em predizer o risco de mortalidade foram investigadas em 1.907 sujeitos com e sem
Risco de Morte
Razões de Chances

Aptidão Cardiorrespiratória

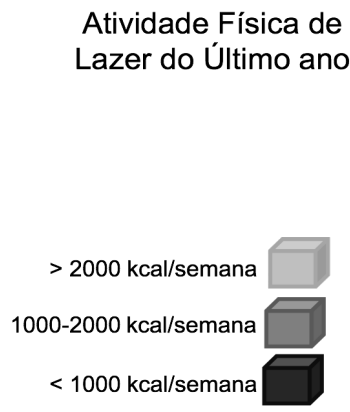

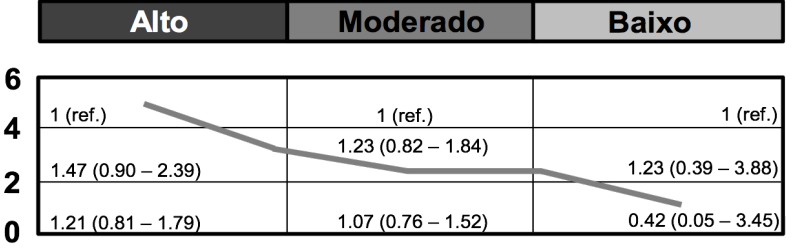

\begin{tabular}{l|l|l|}
$<$ 5.0 METs & $5.0-11.4$ METs & $>11.4$ METs \\
\hline
\end{tabular}

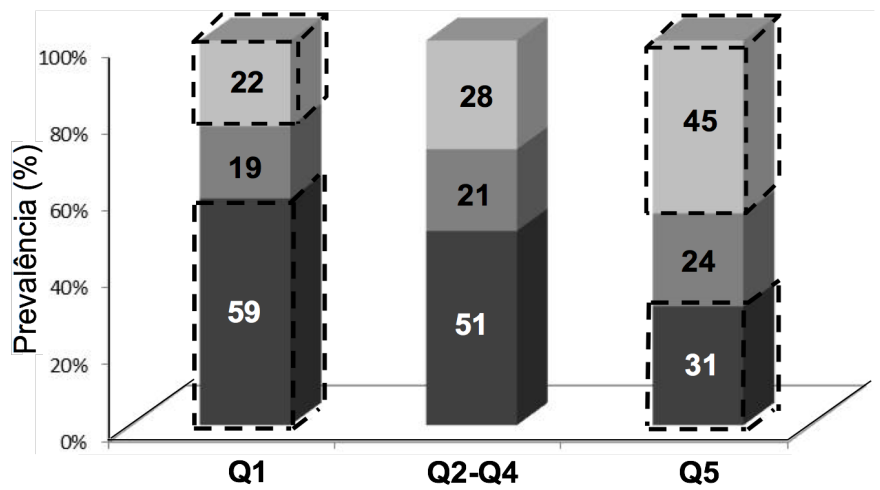

Figura 3. Interação entre aptidão cardiorrespiratória, hábitos de atividade física e mortalidade $\mathrm{Q}=$ quintil. 
doença cardiopulmonar, sendo acompanhados por um tempo médio de $8,7 \pm 5,3$ anos. Todos os indivíduos foram categorizados em grupos em função do gasto calórico de atividades recreativas realizadas no último ano $(<1.000$ $\mathrm{kcal} /$ semana; $1.000-2.000 \mathrm{kcal} / \mathrm{semana} ;>2.000$ $\mathrm{kcal} / \mathrm{semana}$ ) e ainda em função dos seus níveis de aptidão cardiorrespiratória em METs (quintis de 1 a 5), estimados em função da velocidade e inclinação obtidas em teste máximo de esforço. A intensidade relativa foi estimada através de uma escala de percepção subjetiva de esforço. $\mathrm{Na}$ figura 3, podemos observar os resultados das análises combinadas dos grupos. Ao observarmos os indivíduos do primeiro quintil (<5 METs), de baixa aptidão cardiorrespiratória e baixo volume total de atividades realizadas $(<$ $1.000 \mathrm{kcal} / \mathrm{semana}$ ), notamos que, como era de se esperar, $59 \%$ apresentaram risco de mortalidade aproximadamente cinco vezes maior quando comparados ao grupo referência [quintil 5 (11,4 METs) e alto volume (> $2.000 \mathrm{kcal} /$ semana)]. Por outro lado, o que nos chama a atenção é que $22 \%$ dos indivíduos desse grupo continuam apresentando risco elevado, mesmo tendo um volume de atividade física superior a $2.000 \mathrm{kcal} /$ semana. Mais interessante, ao observarmos os indivíduos do quintil 5 , notamos que $31 \%$ não relatam realizar atividades em volume superior a $1.000 \mathrm{kcal} / \mathrm{semana}$ e no entanto continuam apresentando risco reduzido de mortalidade.

Em adendo, quando comparados de modo independente em cada um dos quintis, variações dos volumes de atividade física não apresentaram associações com o risco de mortalidade por todas as causas (valores apresentados no quadro). Nossos resultados colocam em questionamento a associação entre o volume de atividade física e redução de risco de mortalidade. Na medida em que, claramente, maiores níveis de aptidão cardiorrespiratória demonstraram maior associação com a mortalidade do que o volume total realizado nos grupos e que para se alcançar maiores níveis de aptidão cardiorrespiratória, atividades de maior intensidade são necessárias, nossa hipótese é que por maior que seja o volume total despendido, certos indivíduos alcançam tais valores acumulando atividades de leve ou moderada intensidade com maior duração. Para responder a essa hipótese, comparamos a intensidade relativa entre os grupos através da percepção subjetiva de esforço e observamos que, quando comparados aos indivíduos do quinto quintil, a intensidade relativa foi significativamente menor entre os indivíduos do primeiro quintil, não se diferindo entre aqueles que gastavam menos que $1.000 \mathrm{kcal} /$ semana e mais que $2.000 \mathrm{kcal} / \mathrm{semana}$. Uma das limitações do presente estudo, no entanto, é o fato de ter avaliado de modo subjetivo a intensidade relativa das atividades. Por outro lado, devemos considerar que a grande maioria dos estudos observacionais considera apenas a intensidade absoluta das atividades em suas análises, ao classificá-las pelos valores de METs, assumindo, de modo geral, qualquer atividade física com valores superiores a 6 METs como intensa, seja para um indivíduo supostamente sedentário, seja para um melhor condicionado.

Em síntese, até a presente data, são escassos os dados da literatura capazes de estabelecer com clareza a forma da curva de dose-resposta entre a intensidade da atividade física e o risco de mortalidade. Estudos conduzidos em populações que realizaram primariamente atividades de moderada intensidade demonstram existir associação entre atividades moderadas e o risco de mortalidade, presumivelmente em função dos maiores gastos calóricos. No entanto, existe ainda uma carência de dados que possam determinar para dois indivíduos que têm mesmo gasto calórico, se benefícios adicionais seriam alcançados para aquele que despender essa energia realizando atividades de maior intensidade, comparado ao outro indivíduo que realiza o mesmo trabalho exclusivamente com atividade moderada ou até mesmo de leve intensidade. Parte dos resultados por vezes contraditórios na literatura deve-se a algumas limitações inerentes aos estudos observacionais; dentre estas, as dificuldades em se quantificar, 
de modo preciso, os níveis de atividade física, com seus respectivos domínios e componentes, dos indivíduos em situação de vida real. A próxima sessão deste artigo, discutirá um pouco os diversos métodos disponíveis na literatura para se quantificar os hábitos de atividade física.

\section{Métodos de avaliação dos hábitos de atividade física}

A atividade física é um comportamento complexo, e selecionar o instrumento apropriado para a sua avaliação é ainda mais difícil, particularmente entre populações que realizam atividade física ao ar livre ou em ambiente doméstico, onde não possuem um ambiente facilitador de mensuração da atividade. ${ }^{8} \mathrm{~A}$ falta de um método robusto e universal de medida e o uso inconsistente da terminologia 'atividade física' são alguns dos fatores que contribuíram para os resultados conflitantes e de difícil comparação entre os estudos.

O gasto energético é definido como a troca de energia necessária para realizar o trabalho biológico. Componentes que contribuem para o gasto energético total incluem a taxa metabólica basal (engloba de 50 a $70 \%$ da energia total), o efeito térmico do alimento (que contabiliza outros 7-10\%), e a atividade física. Esta última é o componente mais variável do gasto energético total e inclui atividades estruturadas (esportes, lazer, transportes, e atividades de trabalho) e atividades não estruturadas (trabalho doméstico, cuidado de crianças e atividades diárias variadas). ${ }^{28}$

Apesar de similares, os termos 'atividade física' e 'exercício' possuem significados diferentes. A atividade física é definida como qualquer movimento corporal produzido pelos músculos esqueléticos que resulta em gasto energético. E o exercício é definido como um movimento corporal planejado, estruturado e repetitivo, realizado para melhorar ou manter um ou mais componentes da aptidão física. ${ }^{29}$ A atividade física é normalmente quantificada em termos de frequência (dias por semana ou mês), duração (minutos ou horas), intensidade
(METs) e tipo de atividade física realizada (estruturada e não estruturada). $\mathrm{O}$ valor de 1 MET (equivalente a $3,5 \mathrm{ml} / \mathrm{O}_{2} \cdot \mathrm{kg}^{-1} / \mathrm{min}^{-1}$ ), unidade usada para estimar o custo metabólico (consumo de oxigênio) no repouso, também é grosseiramente equivalente a 1 quilocaloria (kcal) por quilograma $(\mathrm{kg})$ de peso corporal por hora $\left(1 \mathrm{kcal} / \mathrm{kg}^{-1} / \mathrm{hora}^{-1}\right) .{ }^{30} \mathrm{O}$ Compêndio de Atividade Física, desenvolvido pela Dra Barbara Ainsworth em 1993 e atualizado nos anos 2000 e 2011, foi desenvolvido tanto para melhorar a comparação dos resultados entre os estudos observacionais que, em sua grande maioria, ainda usam questionários de atividade física, quanto para quantificar o custo energético de uma larga variedade de atividades físicas. ${ }^{31} \mathrm{O}$ gasto energético pode ser quantificado diretamente ou indiretamente através de métodos laboratoriais e de campo. No quadro 1, apresentam-se alguns dos principais métodos de avaliação do gasto calórico e hábitos de atividade física. ${ }^{8}$

Cabe destacar que, apesar de muitos métodos estarem disponíveis para a avaliação do gasto calórico e hábitos de atividade física, a grande maioria destes apresentam limitações quanto ao seu uso em estudos observacionais. Em adendo, cabe notar que, apesar da destacada atenção dada por algumas das mais importantes instituições à elevada prevalência de sedentarismo no mundo, a quantificação da inatividade física tem recebido menos atenção do que a atividade física. Existe uma limitada evidência na literatura atual relacionada à validade e à fidedignidade das medidas que podem ser usadas para avaliar corretamente o comportamento sedentário. ${ }^{32}$

\section{Perspectivas futuras}

Ao longo dos últimos 60 anos, pesquisas de saúde pública têm avaliado os níveis de atividade física para determinar tanto uma associação entre atividade e status de saúde quanto para identificar indivíduos que precisam de intervenção através da atividade física. Atenção inicial deste campo foi dada a atividades 
Quadro 1. Principais métodos de avaliação do gasto calórico e hábitos de atividade física

Método

Carrinhos metabólicos e calorimetria indireta portáti

Água duplamente marcada (ADM)

Bicarbonato marcado

Acelerômetro

Pedômetro

Questionário
Comentários

Método direto mais preciso para se medir o gasto calórico. Condições laboratoriais com o indivíduo em uma câmara hermética. O custo e as limitações técnicas fazem com que a calorimetria direta seja inviável para avaliar hábitos de atividade física relacionada com o gasto calórico em estudos epidemiológicos.

Relações entre o consumo de oxigênio $\left(\mathrm{VO}_{2}\right)$ e o custo calórico da oxidação do substrato. Indivíduos são confinados em uma câmara metabólica durante a avaliação. Com base nas concentrações de gases e taxa de fluxo de ar expirado, o $\mathrm{VO}_{2}$ e o $\mathrm{VCO}_{2}$ podem ser determinados. Devido a restrições da câmara, esta técnica não oferece medidas precisas para o gasto calórico em atividades de campo e por consequência, sua utilização é limitada em estudos epidemiológicos.

Métodos para realização da captação de oxigênio baseado na calorimetria indireta fora da câmara têm sido desenvolvidos. Normalmente, estas técnicas são usadas para avaliar as atividades de tarefas específicas, tais como caminhada/corrida, ciclismo, trabalho doméstico, trabalho de jardinagem e ocupacional. Apesar de usado em estudos epidemiológicos, o custo elevado e a necessidade de treinamento de recursos humanos limitam seu uso.

Uso de marcadores biológicos que refletem a taxa de metabolismo corporal. Utiliza-se da água marcada com isótopos estáveis dosados de acordo com o total de água no corpo. Apesar de fornecer estimativas precisas do gasto calórico em atividades de campo por um período prolongado, uma lacuna importante dessa técnica é não fornecer informações pertinentes a duração, a frequência e a intensidade das atividades. Fornece apenas o gasto calórico total da atividade. Além disso, não é viável em grandes populações devido ao alto custo, disponibilidade dos isótopos e a dificuldade da técnica.

Similar à ADM, tem sido usado para medir o gasto calórico total diário em atividades de campo. As limitações também são similares à $\mathrm{ADM}$, mas pode ser usado para medir o gasto calórico em períodos de tempo menores (dia) comparado à ADM.

Validado em diversas populações, incluindo crianças e idosos. Tem sido usado para validar questionários de atividade física e quantificar associações entre atividade física e diversas doenças. Fornecem informacões sobre a frequência, a duração, a intensidade e os padrões da atividade. Possui a capacidade de armazenar informações durante longos períodos de tempo, porém não é capaz de especificar o tipo de atividade (ex.: caminhar e correr). Acelerômetros tendem a subestimar a atividade relacionada ao gasto energético e não contabilizar o gasto calórico dos membros superiores, atividades aquáticas e atividades em planos inclinados.

Tem demonstrado precisão razoável para o uso em pesquisas em ambientes clínicos onde a caminhada é o principal tipo de atividade. Possui fácil gerenciamento de dados, sendo uma ferramenta de avaliação prática para indivíduos, englobando diferentes populações. Além disso, possui a habilidade em promover mudanças de hábitos de vida e tem sido amplamente usado como uma ferramenta de intervenção. No entanto, não fornece informaç̧ões relacionadas ao tipo, duração e intensidade, e consequentemente não é capaz de quantificar a atividade relacionada ao gasto calórico. Similarmente aos outros monitores de movimento, não tem a capacidade de quantificar movimentos da parte superior do corpo e possui dificuldade na precisão em níveis de atividade física muito baixo.

Método mais usado em estudos epidemiológicos para avaliar níveis de atividade física entre indivíduos que realizam atividades fora do ambiente clínico ou laboratorial. Variam em suas complexidades, tempo e o tipo de atividade avaliada. Comumente validado em indivíduos jovens e saudáveis, pois apresentam limitações em quantificar de modo subjetivo atividades de baixa intensidade, normalmente realizadas por indivíduos menos condicionais, por mulheres e idosos. Por serem em sua grande maioria instrumentos recordatórios, apresentam limitações inerentes ao estimar os hábitos de atividade física e, por consequência, podem apresentar variabilidade grande na estimativa do gasto calórico, principalmente em atividades não estruturadas e de baixa intensidade (comportamentos habituais de trabalho doméstico). de altas intensidades, como esportes, atividades recreacionais ou atividades ocupacionais. A avaliação da atividade física neste tempo era determinada principalmente com questionários, que eram relativamente precisos na observação dessas atividades de alta intensidade. No entanto, avanços tecnológicos associados à vida moderna diminuíram a quantidade de movimentos necessários ao ser humano ao longo do dia, causando uma redefinição do significado de sedentarismo.

$\mathrm{O}$ avanço tecnológico vem aumentando a precisão da avaliação da atividade física, através da qual agora é possível medir simultaneamente movimentos, respostas fisiológicas e características espaciais do movimento humano. É possível também coletar dados eletrônicos quase que em tempo real sobre a 
atividade física utilizando aplicativos usados em computadores, internet e tecnologia de internet sem fio. O desafio dessa nova era de avaliação da atividade física será a incorporação dessas novas tecnologias, bem como o gerenciamento e análise de uma quantidade enorme de dados e em como integrar diferentes tipos de dados que caracterizam os padrões de atividade física relacionados às diversas doenças.

A presente revisão não teve a pretensão de esgotar o tema, mas apenas abordar algumas das questões atuais relevantes que permeiam as discussões no campo da epidemiologia da atividade física, em particular sobre os aspectos metodológicos dos estudos que investigam as relações de dose-resposta entre atividade física e desfechos em saúde.

\section{Referências}

1. Lyons AS, Petrucelli RJ. Medicine: An illustrated history. $1^{\text {st }}$ ed. New York: H. N. Abrams, inc. 1978.

2. U.S. Department of Health and Human Services. Physical activity and health: a report of the Surgeon General. National Center for Chronic Disease Prevention and Health Promotion, editor. Atlanta; 1996.

3. Pate RR, Pratt M, Blair SN, Haskell WL, Macera CA, Bouchard C, et al. Physical activity and public health. A recommendation from the Centers for Disease Control and Prevention and the American College of Sports Medicine. JAMA. 1995 Feb 1;273(5):402-7.

4. Kesaniemi YK, Danforth E Jr, Jensen MD, Kopelman PG, Lefèbvre P, Reeder BA. Doseresponse issues concerning physical activity and health: an evidence-based symposium. Med Sci Sports Exerc. 2001 Jun;33(6 Suppl):S351-8.

5. Physical activity and cardiovascular health. NIH Consensus Development Panel on Physical Activity and Cardiovascular Health. JAMA. 1996 Jul 17;276(3):241-6.

6. Fraser GE, Strahan TM, Sabaté J, Beeson WL, Kissinger D. Effects of traditional coronary risk factors on rates of incident coronary events in a low-risk population. The Adventist Health Study. Circulation. 1992 Aug;86(2):406-13.

7. Simonsick EM, Lafferty ME, Phillips CL, Mendes de Leon CF, Kasl SV, Seeman TE, et al. Risk due to inactivity in physically capable older adults. Am J Public Health. 1993 Oct;83(10):1443-50.

8. Lee IM, editor. Epidemiologic methods in physical activity studies. New York: Oxford University Press; 2009.

9. Bauman AE, Blair SN. Everyone could enjoy the "survival advantage" of elite athletes. BMJ. 2012 Dec 13;345 http://dx.doi. org/10.1136/bmj.e8338.

10. Pocock SJ, Elbourne DR. Randomized trials or observational tribulations? N Engl J Med. 2000 Jun 22;342(25):1907-9. http://dx.doi. org/10.1056/NEJM200006223422511

11. Sesso HD, Gaziano JM, VanDenburgh M, Hennekens CH, Glynn RJ, Buring JE. Comparison of baseline characteristics and mortality experience of participants and nonparticipants in a randomized clinical trial: the Physicians' Health Study. Control Clin Trials. 2002 Dec;23(6):686-702. http://dx.doi. org/S0197245602002350 [pii]

12. Nieman DC. Is infection risk linked to exercise workload? Med Sci Sports Exerc. 2000 Jul;32(7 Suppl):S406-11.

13. Eyre H, Kahn R, Robertson RM, Clark NG, Doyle C, Hong Y, et al. Preventing cancer, cardiovascular disease, and diabetes: a common agenda for the American Cancer Society, the American Diabetes Association, and the American Heart Association. Circulation. 2004 Jun 29;109(25):324455. http://dx.doi.org/10.1161/01. CIR.0000133321.00456.00 http://dx.doi.org/01. CIR.0000133321.00456.00 [pii]

14. Manson JE, Greenland P, LaCroix AZ, Stefanick ML, Mouton CP, Oberman A, et al. Walking compared with vigorous exercise for the prevention of cardiovascular events in women. N Engl J Med. 2002 Sep 5;347(10):716-25. http://dx.doi.org/10.1056/ NEJMoa021067347/10/716 [pii]

15. Kraus WE, Houmard JA, Duscha BD, Knetzger KJ, Wharton MB, McCartney JS, et al. Effects of the amount and intensity of exercise on plasma lipoproteins. N Engl J Med. 2002 Nov 7;347(19):1483-92. http:// dx.doi.org/10.1056/NEJMoa020194347/19/1483 [pii]

16. Saris WH, Blair SN, van Baak MA, Eaton SB, Davies PS, Di Pietro L, et al. How much physical activity is enough to prevent unhealthy weight gain? Outcome of the IASO 1st Stock Conference and consensus statement. Obes Rev. 2003 May;4(2):101-14.

17. Morris JN, Heady JA, Raffle PAB, Roberts CG, Parks JW. Coronary heart disease and 
physical activity of work. Lancet. 1953 Nov 28;262(6796):1111-20.

18. Lee Y. The predictive value of self assessed general, physical, and mental health on functional decline and mortality in older adults. J Epidemiol Community Health. 2000 Feb;54:123-9.

19. Sesso HD, Paffenbarger RS J, Lee IM. Physical activity and coronary heart disease in men: The Harvard Alumni Health Study. Circulation. 2000 Aug 29;102(9):975-80.

20. Lee IM, Sesso HD, Paffenbarger RS Jr. Physical activity and coronary heart disease risk in men: does the duration of exercise episodes predict risk? Circulation. 2000 Aug 29;102(9):981-6.

21. Hoffmeister H, Mensink GB, Stolzenberg H, Hoeltz J, Kreuter H, Laaser U, et al. Reduction of coronary heart disease risk factors in the German cardiovascular prevention study. Prev Med. 1996 Mar-Apr;25(2):135-45. http:// dx.doi.org/S0091743596900390 [pii]

22. Kushi LH, Fee RM, Folsom AR, Mink PJ, Anderson KE, Sellers TA. Physical activity and mortality in postmenopausal women. JAMA. 1997 Apr 23-30;277(16):1287-92.

23. Rakowski W, Mor V. The association of physical activity with mortality among older adults in the Longitudinal Study of Aging (1984-1988). J Gerontol. 1992 Jul;47(4):M122-9.

24. Willet W. Nutritional epidemiology. 2nd ed. New York: Oxford University Press; 1998.

25. Tanasescu M, Leitzmann MF, Rimm EB, Willett WC, Stampfer MJ, Hu FB. Exercise type and intensity in relation to coronary heart disease in men. JAMA. 2002 Oct
23-30;288(16):1994-2000. http://dx.doi.org/ joc20649 [pii]

26. Lee IM, Hsieh CC, Paffenbarger RS Jr. Exercise intensity and longevity in men. The Harvard Alumni Health Study. JAMA. 1995 Apr 19;273(15):1179-84.

27. Lollgen H, Bockenhoff A, Knapp G. Physical activity and all-cause mortality: an updated meta-analysis with different intensity categories. Int J Sports Med. 2009 Mar;30(3):213-24. http://dx.doi. org/10.1055/s-0028-1128150

28. Ravussin E, Bogardus C. A brief overview of human energy metabolism and its relationship to essential obesity. Am J Clin Nutr. 1992 Jan;55(1 Suppl):242S-5S.

29. Caspersen CJ, Powell KE, Christenson GM. Physical activity, exercise, and physical fitness: definitions and distinctions for healthrelated research. Public Health Rep. 1985 Mar-Apr;100(2):126-31.

30. Taylor HL, Jacobs DR Jr, Schucker B, Knudsen J, Leon AS, Debacker G. A questionnaire for the assessment of leisure time physical activities. J Chronic Dis. 1978;31(12):741-55.

31. Ainsworth BE, Haskell WL, Herrmann SD, Meckes N, Bassett DR, Jr., Tudor-Locke C, et al. 2011 Compendium of Physical Activities: a second update of codes and MET values. Med Sci Sports Exerc. 2011 Aug;43(8):1575-81. http://dx.doi.org/10.1249/ MSS.0b013e31821ece12

32. Atkin AJ, Gorely T, Clemes SA, Yates T, Edwardson C, Brage S, et al. Methods of Measurement in epidemiology: sedentary Behaviour. Int J Epidemiol. 2012 Oct;41(5):1460-71. http://dx.doi.org/dys118 [pii] 


\section{Autores}

\section{Ada Fernanda P. S. Lima}

Laboratório de Atividade Física e Promoção da Saúde. Instituto de Educação Física e Desportos. Universidade do Estado do Rio de Janeiro. Rio de Janeiro, RJ, Brasil.

\section{Alexandre H. Okano}

Departamento de Educação Física. Centro de Ciências da Saúde. Universidade Federal do Rio Grande do Norte. Natal, RN, Brasil.

\section{Alice R. Sampaio}

Laboratório de Atividade Física e Promoção da Saúde. Instituto de Educação Física e Desportos. Universidade do Estado do Rio de Janeiro. Rio de Janeiro, RJ, Brasil.

\section{Ana Paula M. Guttierres}

Laboratório de Atividade Física e Promoção da Saúde. Instituto de Educação Física e Desportos. Universidade do Estado do Rio de Janeiro. Rio de Janeiro, RJ, Brasil.

\section{Astrogildo V. Oliveira Júnior}

Departamento de Educação Física e Folclore. Colégio Pedro II. Rio de Janeiro, RJ, Brasil.

\section{Brenno S. Silva}

Programa de Pós-graduação em Ciências da Atividade Física. Universidade Salgado de Oliveira. Niterói, RJ, Brasil.

\section{Daniel A. Bottino}

Laboratório de Pesquisas Clínicas e Experimentais em Biologia Vascular. Centro Biomédico.

Universidade do Estado do Rio de Janeiro. Rio de Janeiro, RJ, Brasil.

\section{Dionizio Mendes Ramos Filho}

Laboratório de Bioenergética e Fisiologia Mitocondrial. Centro de Ciências da Saúde. Universidade Federal do Rio de Janeiro. Rio de Janeiro, RJ, Brasil.

\section{Elirez B. Silva}

Laboratório de Pesquisa Clínica Escola. Departamento de Fisioterapia. Universidade Gama Filho. Rio de Janeiro, RJ, Brasil.

\section{Ercole C. Rubini}

Laboratório de Fisiologia do Exercício. Universidade Estácio de Sá. Rio de Janeiro, RJ, Brasil.

\section{Fabrício V. A. Vasconcellos}

Centro de Investigação, Formação, Inovação, Intervenção e Desporto. Faculdade de Desporto. Universidade do Porto. Porto, Portugal.

\section{Felipe A. da Cunha}

Programa de Pós-graduação em Ciências Médicas. Universidade do Estado do Rio de Janeiro. Rio de Janeiro, RJ, Brasil.

\section{Fernanda Monteiro}

Laboratório de Atividade Física e Promoção da Saúde. Instituto de Educação Física e Desportos. Universidade do Estado do Rio de Janeiro. Rio de Janeiro, RJ, Brasil.

\section{Flávia Porto}

Programa de Pós-graduação em Ciências do Exercício e do Esporte. Universidade Gama Filho. Rio de Janeiro, RJ, Brasil. 


\section{Gustavo C. Lopes}

Laboratório de Atividade Física e Promoção da Saúde. Instituto de Educação Física e Desportos. Universidade do Estado do Rio de Janeiro. Rio de Janeiro, RJ, Brasil.

\section{Jonas L. Gurgel}

Programa de Pós-graduação em Ciências

Cardiovasculares. Universidade Federal Fluminense.

Niterói, RJ, Brasil.

\section{Jonathan Myers}

VA Palo Alto Health Care System. Cardiology Division. Stanford University. Palo Alto, California, United States.

\section{Karynne Grutter}

Laboratório de Anatomia Humana. Universidade Castelo Branco. Rio de Janeiro, RJ, Brasil.

\section{Lenifran Matos-Santos}

Programa de Pós-graduação em Ciências da Atividade Física. Universidade Salgado de Oliveira. Niterói, RJ, Brasil.

\section{Lorena Paes}

Programa de Pós-graduação em Fisiopatologia Clínica e Experimental. Universidade do Estado do Rio de Janeiro. Rio de Janeiro, RJ, Brasil.

\section{Luciane P. da Costa}

Laboratório de Atividade Física e Promoção da Saúde. Instituto de Educação Física e Desportos. Universidade do Estado do Rio de Janeiro. Rio de Janeiro, RJ, Brasil.

\section{Luiz G. Kraemer-Aguiar}

Departamento de Medicina Interna. Faculdade de Ciências Médicas. Universidade do Estado do Rio de Janeiro. Rio de Janeiro, RJ, Brasil.

\section{Matheus R. Hausen}

Laboratório de Atividade Física e Promoção da Saúde. Instituto de Educação Física e Desportos. Universidade do Estado do Rio de Janeiro. Rio de Janeiro, RJ, Brasil.

\section{Paulo T. V. Farinatti}

Laboratório de Atividade Física e Promoção da Saúde. Instituto de Educação Física e Desportos. Universidade do Estado do Rio de Janeiro. Rio de Janeiro, RJ, Brasil.

\section{Rafael A. Montenegro}

Programa de Pós-graduação em Fisiopatologia Clínica e Experimental. Universidade do Estado do Rio de Janeiro. Rio de Janeiro, RJ, Brasil.

\section{Raul A. Freire}

Programa de Pós-graduação em Ciências da Atividade Física. Universidade Salgado de Oliveira. Niterói, RJ, Brasil.

\section{Renato O. Massaferri}

Programa de Pós-graduação em Ciências da Atividade Física. Universidade Salgado de Oliveira. Niterói, RJ, Brasil.

\section{Ricardo B. Oliveira}

Laboratório de Atividade Física e Promoção da Saúde. Instituto de Educação Física e Desportos. Universidade do Estado do Rio de Janeiro. Rio de Janeiro, RJ, Brasil.

\section{Ricardo G. Cordeiro}

Programa de Pós-graduação em Ciências da Atividade Física. Universidade Salgado de Oliveira. Niterói, RJ, Brasil.

\section{Sérgio Machado}

Programa de Pós-graduação em Ciências da Atividade Física. Universidade Salgado de Oliveira. Niterói, RJ, Brasil.

\section{Taciana Pinto}

Laboratório de Atividade Física e Promoção da Saúde. Instituto de Educação Física e Desportos. Universidade do Estado do Rio de Janeiro. Rio de Janeiro, RJ, Brasil.

\section{Tainah P. Lima Monteiro}

Programa de Pós-graduação em Ciências Médicas. Faculdade de Ciências Médicas. Universidade do Estado do Rio de Janeiro. Rio de Janeiro, RJ, Brasil.

\section{Tânia M. P. F. Paschoalino}

Hospital Universitário Antônio Pedro. Universidade Federal Fluminense. Niterói, RJ, Brasil.

\section{Walace D. Monteiro}

Laboratório de Atividade Física e Promoção da Saúde. Instituto de Educação Física e Desportos. Universidade do Estado do Rio de Janeiro. Rio de Janeiro, RJ, Brasil. 